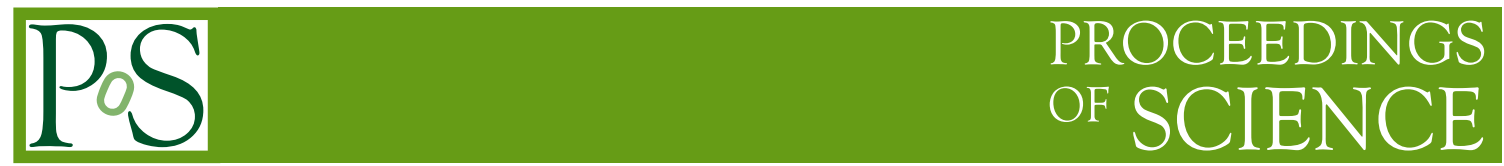

\title{
Hadronic Calibration for the ATLAS Jet Trigger
}

\section{N. Anjos ${ }^{* \dagger}$ and P. Conde Muíño}

Laboratório de Instrumentação e Física Experimental de Partículas (LIP), Lisboa, Portugal

\section{Aracena}

Stanford Linear Accelerator Center (SLAC), Stanford, USA

\section{Santamarina Rios and M. Schram}

Department of Physics, McGill University, Montreal, Canada

The ATLAS trigger system must reduce the $40 \mathrm{MHz}$ event rate delivered by the LHC to $200 \mathrm{~Hz}$. This is done by selecting online high $p_{T}$ objects. In proton-proton collisions, the main background for jets are also jets. Thus, the main difficulty for the jet trigger is to accurately measure the jet energy, in order to distinguish high $p_{T}$ jets from low $p_{T}$ ones. The measurement of the jet energy scale is a complex issue, specially for non-compensating hadronic calorimeters, like the ATLAS one. This paper describes the strategies used to perform the hadronic calibration for the ATLAS jet trigger.

Physics at LHC 2008

29 September - October 4, 2008

Split, Croatia

\footnotetext{
*Speaker.

${ }^{\dagger}$ Work supported by FCT through grant SFRH/BPD/37964/2007.
} 


\section{Introduction}

ATLAS [1] is a multipurpose experiment at the Large Hadron Collider (LHC). By design, the LHC will provide proton-proton collisions at a centre-of-mass energy of $14 \mathrm{TeV}$, a luminosity of $10^{34} \mathrm{~cm}^{-2} \mathrm{~s}^{-1}$ and a bunch crossing rate of $40 \mathrm{MHz}$. The resulting volume of data is too large to be stored. The trigger system has to select online both expected and unexpected physically interesting events, and only these will be recorded. The ATLAS trigger system does this by selecting high $p_{T}$ objects like muons, electrons, jets, taus and large missing transverse energy. It is divided in three levels. The first one (L1) is hardware based, with coarse granularity and $2 \mu$ s latency. The Second Level Trigger (L2) and the Event Filter (EF, third level trigger) are software based. The L2 has a maximum processing time of the order of $40 \mathrm{~ms}$ and has to reduce the rate from $75 \mathrm{KHz}$ to $2 \mathrm{KHz}$, while the EF can run more developed algorithms to reduce the rate to $200 \mathrm{~Hz}$. Jointly, L2 and EF constitute the High Level Trigger (HLT).

In hadronic colliders, the main background for a jet trigger are also jets. Therefore, it is crucial to accurately measure the jet energy, in order to distinguish high $p_{T}$ jets from low $p_{T}$ ones. The measurement of the jet energy scale is a complex process which requires good understanding of several effects. One example is the fact that the ATLAS calorimeter is non-compensating, meaning that the response to the electromagnetic (EM) component of a hadron shower is not equal to the response to the non-EM component. This and other effects are accounted for through calibration. In ATLAS, the procedure to calibrate both the trigger and the offline jets, uses the Monte Carlo (MC) simulation in order to extract the calibration constants.

\section{The ATLAS Jet Trigger at L2}

The algorithm for L2 jets [2] accesses EM and hadronic calorimeter data that lies in a rectangular region in $(\eta, \phi)$, centered around a "Region of Interest" (RoI) defined by the the L1 jet, which acts as seed. After data unpacking, a grid of the detector elements within the region is build. By default the full granularity is used, and each calorimeter cell constitutes a detector element. To speed up data unpacking, it is also possible to read only the energy sums of all the cells belonging to a Front-End Buffer (FEB). This results in a algorithm which is 3 times faster while achieving similar performance. The L2 trigger jets are then build using an iterative cone algorithm. The algorithm loops within the grid and calculates the energy weighted $(\eta, \phi)$ position of the detector elements inside a cone of fixed radius $R=\sqrt{(\Delta \eta)^{2}+(\Delta \phi)^{2}}$ around the seed. A fast calibration algorithm is applied, as described in Sec. 3 . The outcome of this algorithm is a jet defined by its $(\eta, \phi)$ position and total energy. The L2 jets are accepted by the trigger if the energy of the jet candidate is above some predefined $E_{T}$ threshold.

\section{Calibration of the L2 Jets}

After jet building at L2, the calorimeter energy scale is set at the EM scale, which does not provide an accurate measure of the jet energy. The L2 jet calibration [2] is based on the Sampling method [3]. For calibration purposes, a jet is regarded as composed of energy deposits in calorimeter samplings. The calibrated jet energy can then be written as $E_{\mathrm{jet}}^{\mathrm{rec}}=\sum_{i} w_{i} E_{i}$, where $E_{i}$ is 
the uncalibrated energy in the sampling $i$, and the sum runs over all samplings considered. The $w_{i}$ weights are calculated using MC samples, by minimizing the function

$$
\chi^{2}=\sum_{m=1}^{N_{\mathrm{jets}}}\left[\frac{\left(E_{m}^{\mathrm{truth}}-E_{m}^{\mathrm{rec}}\right)}{\sigma_{m}}\right]^{2},
$$

where the sum run over all jets in the events. The true energy of the $m$-th jet in the event is labeled $E_{m}^{\text {truth }}$ and is obtained from the MC truth information, running a cone jet algorithm with radius $\Delta R=0.4$ over the MC truth particles and finding the truth jet that is closest to the $m$-th jet. The weights are chosen to be energy dependent and parametrized by $w_{i}=a+b \log (E)$, where $E$ is the jet energy. The procedure is validated by comparing the calibrated jet energy with the MC truth jet energy. After calibration, the jet energy scale is correct within $2 \%$ for all energies (Fig. 1), and the jet energy resolution is improved by a few $\%$ [2].
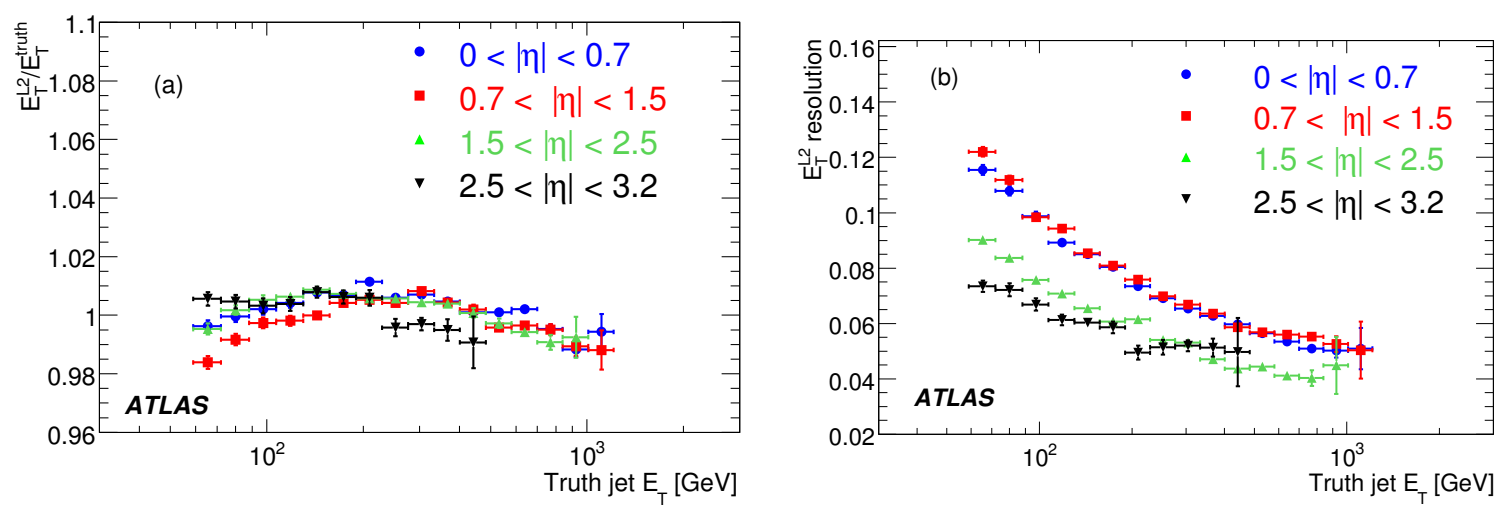

Figure 1: Jet energy scale for the L2 jets as a function of the truth jet $E_{T}$ (a). Jet energy resolution as a function of the truth energy of the jet (b). The results are obtained for four different bins in $\eta$ and after calibration.

\section{Conclusions}

The ATLAS L2 jet trigger uses a cone algorithm to reconstruct jets and applies a simple calibration procedure. Calibration weights depend on the jet energy and $\eta$, and are calculated using MC. After calibration, the L2 jet energy scale is correct within $2 \%$ and energy resolution is improved. Gains in speed are possible using coarser granularity with similar performance. In the future, in-situ calibration with data should be used to validate the MC based calibration.

\section{References}

[1] ATLAS Collaboration, G Aad et al, The ATLAS Experiment at the CERN Large Hadron Collider, JINST 3 S08003 (2008)

[2] ATLAS Collaboration, G Aad et al, Expected Performance of the ATLAS Experiment - Detector, Trigger and Physics, CERN-OPEN-2008-020 (2008)

[3] A. Gupta, F. Merrit and J Proudfoot, Jet Energy Correction Using Longitudinal Weighting, CERN-ATL-COM-PHY-2006-062 (2006) 